\title{
MODERN METHODS OF PATIENTS TREATMENT WITH DENTITION DEFECTS COMBINED WITH FUNCTIONAL DISORDERS OF THE TEMPOROMANDIBULAR JOINT
}

10.36740/WLek202010126

\author{
Roman Ozhohan, Mykola M. Rozhko, Zinoviy R. Ozhogan, Roman M. Khopta, Lidiia Miziuk \\ IVANO-FRANKIVSK NATIONAL MEDICAL UNIVERSITY, IVANO-FRANKIVSK, UKRAINE
}

\begin{abstract}
The aim: The purpose of the research was to increase the efficiency of treatment at patients with dentition defects and functional disorders of the temporomandibular joint. Materials and methods: We examined 140 patients aged from 25 to 65 years with functional disorders of the temporomandibular joint, analysed there diagnostic models in the articulator and 3D scanner, used radiographic methods of examination and condylography.

Results: The results of the conducted studies showed that in the 1st group, the biomechanical parameters of temporomandibular joint movements before treatment were significantly lower: $6,4 \pm 0,2 \mathrm{~mm}$ and $6,8 \pm 0,2 \mathrm{~mm}$ for right and left temporomandibular joint during protrusion movements; 7,8 $\pm 0,1 \mathrm{~mm}$ and 7,9 $\pm 0,2 \mathrm{~mm}$ during vertical movements; $6,7 \pm 0,1 \mathrm{~mm}$ and $6,5 \pm 0,2 \mathrm{~mm}$ during transversal movements. After 12 month of complex and prosthetic treatment with a help of non-removable dentures the magnitude of movements remains at the achieved level at all excursions. As a result, proposed by us comprehensive treatment of functional disorders of the temporomandibular joint is carried out in stages. The proposed design of an individual tire makes it possible to gradually increase the bite height and perfectly forms the occlusal ratio. After 6 and 12 months of the proposed treatment, we have found that the indicators of vertical and protrusion movements of articular heads at patients of 2nd group were significantly better than before treatment.

Conclusions: The proposed design of an individual tire makes it possible to gradually increase the bite height and perfectly forms the occlusal ratio.
\end{abstract}

KEY WORDS: temporomandibular joint, dentition defects, individual splint, condylography, dentures

Wiad Lek. 2020;73(10):2241-2245

\section{INTRODUCTION}

Currently, there is a significant prevalence of dentition defects associated with dental defects and temporomandibular joint (TMJ) disorders, which in turn can lead to headache, joint pain, stiffness and limited movements of the mandible, irradiation of pain to the ear, temple, hearing loss, as well as a feeling of tightness and pain of the masticatory muscles at the end of the day $[1,2]$.

According to the literature, TMJ diseases at different stages occur in $25-65 \%$ of the world population, in particular among adolescents (young people), this figure ranges from $16-30 \%[3,4,5]$. The difficulties in diagnosing TMJ diseases is also due to the lack of a sufficiently clear radiological characteristics of the constituent elements of TMJ, due to anatomical features of the joint structures and the close location of a skull base $[6,7]$.

Landa J.S. [8] noted that the masticatory muscles must be in constant harmony with the state of teeth occlusion and TMJ structure. Campbell L. [9] argued that muscle pain occurs during vasospasm, chills, lack of oxygen, acidosis, and fatigue. Buman A.N. and co-authors $[10,11]$ presented data on adaptive and progressive mechanisms of TMJ tissues, which is a separate type of biological microsystem, highlighting the surface of joint cavity, disc, bilaminar zone as a separate stabilizing mechanism, the function of which is to ensure stable position of lower head of the mandibular in the articular fossa at different phases of it's movement.

Nowadays, three main theories of TMJ dysfunction are considered: occlusal violations, muscle imbalance, psychophysiological phenomenon. Khvatova V.A., Novikov V.M. [10] identified two emerging factors: physical, emotional content and occlusal disharmony. An interaction of these factors can lead to a hyperfunction of masticatory muscles which in turn leads to pain and reduction of joint movement.

Okeson J. considers psychoemotional factor to be one of the main etiological factor among all temporomandibular disorders. The examination results of our patients show the dominance of muscular component at functional disorders of the masticatory system in combination with myofascial pain. According to the author less significant is the element of occlusion (about $20 \%$ of etiological factors) [12, 13, 14].

Ribalov O.V. $[15,16]$ has shown experimentally that extended muscles contraction under the emotional stress can lead to pain. The author believes that the ongoing reduction of the masticatory capacity can be connected with the inflammatory process, irritation of the skull, the upper segment of spine and tooth. Hence, the most quickly reduction could be achieved under emotional stress.

Occlusal disorders reflexively can lead to muscle tension and spasm, causing the development of TMJ dysfunction 
and pain. The study of a neuro-muscular pathology combined with temporomandibular disorders and peculiarities of its manifestations were considered in such studies [5, $9,12,17]$. Topographic-anatomic and clinical-radiological examinations were performed in such studies $[11,18,19]$.

Nowadays, the biggest part of foreign experts supports the concept of a multifactorial theory of TMJ dysfunction, which is based on the instability of the masticatory system $[8,20,21,22]$.

The presence of dentition defects at patients leads to violations of occlusal relations, development of dental deformations and finally progression of periodontal disease $[1,4,24]$. Quite often, it can be admitted a progression of functional disorders in the temporomandibular joint after direct or indirect restorations, especially the lateral group of teeth or after prosthetic treatment using non-removable dentures or partially removable dentures $[13,19]$.

We should pay great attention to functional disorders of the TMJ that can be achieved after incorrect occlusal ratios of fixed dentures or removable dentures. Moreover, it was proposed a numerous variety of modern methods of prosthetic treatment that could eliminate functional disorders of the TMJ with the help of special tires $[1,2,6,23]$.

Therefore, as you can see an individual approach of restoring the integrity of dentition not only solves the compliance of dentures to the needs of patients, but also allows to prevent the development of the dysfunction of TMJ and masticatory muscles.

\section{THE AIM}

To increase the efficiency of treatment at patients with functional disorders of temporomandibular joint and dentition defects with a help of clinical and condylographic justification of the proposed method.

\section{MATERIALS AND METHODS}

Firstly, we have examined patients with functional disorders of the temporomandibular joint combined with dentition defects. Secondly, we have analyzed diagnostic models in the articulator and 3D scanner. Finally, we have used orthopantomography and computed tomography, as well as condylography. During the research we have examined 140 patients aged from 25 to 65 years, who according to clinical features and treatment methods were divided into the following groups:

1 st group - patients with functional disorders of the TMJ (thyroid dysfunction), dentition defects, examined and treated by conventional methods (60 people).

2nd group - patients with functional disorders of the TMJ, dentition defects, examined and treated by the proposed methods using individual tire, articulation system "Cadiax Compact" and 3D scanner (60 people).

Only 20 patients had intact dentition and no signs of TMJ dysfunction.

We have proposed a method of manufacturing an individual tire for eliminating functional disorders of the temporomandibular joint. Furthermore, comprehensive functional disorders treatment of the temporomandibular joint that was proposed by us was carried out in stages, which included the diagnosis of occlusal relationships, selective grinding of occlusal surfaces and condylography. Aqualizer was used for 3-4 weeks after which temporary dentures were made to replace dentition defects, then we used individual tire. Permanent prosthetics were performed with aesthetic non-removable dentures after elimination of TMJ dysfunction, on average 3-6 months after the start of treatment.

\section{RESULTS AND DISCUSSION}

To substantiate the proposed comprehensive treatment at patients with dentition defects and dysfunction of the temporomandibular joint we have recorded the biomechanical parameters of the mandible articular heads movements.

The comparison of TMJ biomechanical parameters before and after non-removable dentures with the usage of an individual tire was performed. The results of the biomechanical parameters study at patients during protrusion movements at right and left TMJ were 10,6 $\pm 0,5 \mathrm{~mm}$ and $9,5 \pm 0,4 \mathrm{~mm}$, respectively, during vertical movements $12,02 \pm 0,2 \mathrm{~mm}$ and $11,4 \pm 0,3 \mathrm{~mm}$, during transverse movements $-10,5 \pm 0,3 \mathrm{~mm}$ and $10,2 \pm 0,3 \mathrm{~mm}$.

The results of the biomechanical parameters study at patients with dentition defects before and after a comprehensive conventional treatment and prosthetics are presented in table 1 . We established a syndrome of temporomandibular joint dysfunction at all patients for whom we recorded individual biomechanical parameters of TMJ with a help of "Cadiax Compact".

Analyzing the obtained results, we have found that in the examined group of patients with dentition defects, the biomechanical parameters at all planes are significantly reduced compared to the norm.

At 1st group of patients, the biomechanical parameters of TMJ movements before treatment were significantly lower than normal: during protrusion movements - 6,4 \pm $0,2 \mathrm{~mm}$ and $6,8 \pm 0,2 \mathrm{~mm}$ for right and left TMJ; during vertical movements $-7,8 \pm 0,1 \mathrm{~mm}$ and $7,9 \pm 0,2 \mathrm{~mm}$, respectively; during transverse movements $-6,7 \pm 0,1 \mathrm{~mm}$ and 6,5 $\pm 0,2 \mathrm{~mm}$ (Tables 1,2).

In 6 months after complex and prosthetic treatment by means of non-removable dentures the magnitude of mandible protrusion movements considerably increases ( $\mathrm{p}<0,05)$ up to $7,4 \pm 0,2 \mathrm{~mm}$ and $7,7 \pm 0,2 \mathrm{~mm}$ in the right and left TMJ. In addition, a magnitude of vertical movements of the articular heads leaves up to $8,1 \pm 0,2$ $\mathrm{mm}$ and $8,2 \pm 0,2 \mathrm{~mm}$ and during mandible movements to the right and left up to $7,1 \pm 0,3 \mathrm{~mm}$ and $7,3 \pm 0,1 \mathrm{~mm}$, respectively $(\mathrm{p}<0,05)$.

After 12 months of complex and prosthetic treatment of patients with non-removable dentures, the magnitude of protrusion movements of the mandible remains at the achieved level and was 7,6 $\pm 0,2 \mathrm{~mm}$ and 7,8 $\pm 0,2 \mathrm{~mm}$ in the right and left TMJ, respectively. Moreover, the indicators 
Table I. Biomechanical parameters of the articular head movements (TMJ) in the sagittal and vertical planes at patients of $1^{\text {st }} \mathrm{group}$, ( $\mathrm{M} \pm \mathrm{m}, \mathrm{mm}$ )

\begin{tabular}{cccccc}
\hline \multirow{2}{*}{ Terms of observation } & \multicolumn{2}{c}{ Protrusion, $\mathbf{m m}$} & \multicolumn{3}{c}{ Vertical, mm } \\
\cline { 2 - 6 } & right & left & right & left \\
\hline Before treatment & $6,4 \pm 0,2$ & $6,8 \pm 0,2$ & $7,8 \pm 0,1$ & $7,9 \pm 0,2$ \\
\hline After 6 month & $7,4 \pm 0,2^{*}$ & $7,7 \pm 0,2^{*}$ & $8,1 \pm 0,2$ & $8,2 \pm 0,2$ \\
\hline After 12 month & $7,6 \pm 0,2^{*}$ & $7,8 \pm 0,2^{*}$ & $8,3 \pm 0,2^{*}$ & $8,2 \pm 0,2^{*}$ \\
\hline
\end{tabular}

* $-p<0.05$, compared with pre-treatment

Table II. Biomechanical parameters of the articular head movements (TMJ) in the transverse plane at patients of $1^{\text {st }}$ group, $(\mathrm{M} \pm \mathrm{m}, \mathrm{mm})$

\begin{tabular}{ccc}
\hline Terms of observation & Meziotrusion, mm & Mediotrusion,mm \\
\hline Before treatment & $6,7 \pm 0,1$ & $6,5 \pm 0,2$ \\
\hline After 6 month & $7,1 \pm 0,3$ & $7,3 \pm 0,1^{*}$ \\
\hline After 12 month & $7,2 \pm 0,3^{*}$ & $7,5 \pm 0,1^{*}$ \\
\hline
\end{tabular}

* $-p<0.05$, compared with pre-treatment

Table III. Biomechanical parameters of the articular head movements (TMJ) in the sagittal and vertical planes at patients of the $2^{\text {nd }} \mathrm{group},(\mathrm{M} \pm \mathrm{m}, \mathrm{mm})$

\begin{tabular}{ccccc}
\hline \multirow{2}{*}{ Terms of observation } & \multicolumn{2}{c}{ Protrusion, $\mathbf{m m}$} & \multicolumn{2}{c}{ Vertical, mm } \\
\cline { 2 - 5 } & right & left & right & left \\
\hline Before treatment & $6,3 \pm 0,3$ & $6,7 \pm 0,4$ & $7,5 \pm 0,4$ & $7,8 \pm 0,3$ \\
\hline After tire & $7,5 \pm 0,3^{*}$ & $7,9 \pm 0,3^{*}$ & $8,4 \pm 0,3^{*}$ & $8,6 \pm 0,3^{*}$ \\
\hline After 6 month & $8,6 \pm 0,2^{*}$ & $8,5 \pm 0,3^{*}$ & $8,7 \pm 0,3^{*}$ & $8,9 \pm 0,2^{*}$ \\
\hline After 12 month & $8,9 \pm 0,2^{*}$ & $9,0 \pm 0,3^{*}$ & $9,4 \pm 0,3^{*}$ & $9,5 \pm 0,2^{*}$ \\
\hline
\end{tabular}

* $-p<0.05$, compared with pre-treatment

Table IV. Biomechanical parameters of the articular head movements (TMJ) in the transverse plane at patients of the $2^{\text {nd }}$ group, $(\mathrm{M} \pm \mathrm{m}, \mathrm{mm})$

\begin{tabular}{ccc} 
Terms of observation & Mediotrusion to the right, $\mathbf{~ m m}$ & Mediotrusion to the left, $\mathbf{m m}$ \\
\hline Before treatment & $6,6 \pm 0,4$ & $6,9 \pm 0,5$ \\
\hline After tire & $8,1 \pm 0,3^{*}$ & $7,8 \pm 0,4^{*}$ \\
\hline After 6 month & $8,5 \pm 0,2^{*}$ & $8,3 \pm 0,4^{*}$ \\
\hline After 12 month & $8,8 \pm 0,2^{*}$ & $8,9 \pm 0,4^{*}$ \\
\hline
\end{tabular}

* $-p<0.05$, compared with pre-treatment

of the magnitude of the articular heads movements in the vertical plane were maintained at the same level $-8,3 \pm 0,2$ $\mathrm{mm}, 8,2 \pm 0,2 \mathrm{~mm}$ and the same with movements of the lower jaw to the left and right $-7,2 \pm 0,3 \mathrm{~mm}$ and 7,5 $\pm 0,1 \mathrm{~mm}$.

At patients of the 2 nd group, who received the proposed complex treatment, the biomechanical parameters of the TMJ movements before treatment were significantly lower $(\mathrm{p}<0,05)$ and the magnitude of protrusion movements indicates $6,3 \pm 0,3 \mathrm{~mm}$ and $6,7 \pm 0,4 \mathrm{~mm}$ for the right and left joints (Tables 3, 4).

After application of the proposed tire for 8-12 weeks, these figures increased significantly to 7,5 $\pm 0,3 \mathrm{~mm}$ and 7,9 $\pm 0,3$ $\mathrm{mm}$, respectively $(\mathrm{p}<0,05)$. After 6 months of the proposed comprehensive and prosthetic treatment at patients with non-removable dentures the magnitude of the mandible protrusion movements was significantly higher than before treatment and indicated 8,6 $\pm 0,2 \mathrm{~mm}$ and $8,5 \pm 0,3 \mathrm{~mm}$ in the right and left TMJ, respectively. After 12 months of treatment, these figures were slightly improved: $8,9 \pm 0,2$ $\mathrm{mm}$ and 9,0 $\pm 0,3 \mathrm{~mm}$. During vertical movements, the biomechanical parameters at patients of 2 group were 7,5 \pm
$0,4 \mathrm{~mm}$ and 7,8 $\pm 0,3 \mathrm{~mm}$ for the right and left TMJ before treatment. After application of the proposed splint these indicators significantly increased at that group of patients to $8,4 \pm 0,3 \mathrm{~mm}$ and $8,6 \pm 0,3 \mathrm{~mm}$ and in 6 months indicated $8,7 \pm 0,3 \mathrm{~mm}$ and $8,9 \pm 0,2 \mathrm{~mm} .12$ months after treatment, the studied parameters were significantly better than previous one: $9,4 \pm 0,3 \mathrm{~mm}$ and $9,5 \pm 0,2 \mathrm{~mm}$. The indicators at patients of 2 nd group during transverse movements of a mandible before treatment were $6,6 \pm 0,4 \mathrm{~mm}$ for the right and 6,9 $\pm 0,5$ for the left TMJ. In this group of patients after the usage of the proposed tire these indicators significantly increased to $8,1 \pm 0,3 \mathrm{~mm}$ and $7,8 \pm 0,4 \mathrm{~mm}$, respectively $(\mathrm{p}<0,05) .6$ months after orthopedic treatment, stabilization and improvement of these parameters at the level of $8,5 \pm$ $0,2 \mathrm{~mm}$ and $8,3 \pm 0,4 \mathrm{~mm}$ for the right and left TMJ can be noted. 12 months after the proposed treatment at patients of the 2 nd group the studied parameters were better than previous one and indicated $8,8 \pm 0,2 \mathrm{~mm}$ and $8,9 \pm 0,4 \mathrm{~mm}$.

When examining patients of the control group with intact dentition using articulation system "Cadiax Compact", we established the qualitative characteristics of TMJ 
biomechanical parameters. In particular, the excursion and incursion movements of the articular heads overlapped with each other at all examined patients during protrusion, mediotrusion and vertical displacement of the mandible. We studied and analyzed the indicators of the articular pathway, namely the symmetry of movements at the right and left TMJ during displacement of the mandible at sagittal and vertical planes. At the examined group of patients, protrusion and vertical movements of the mandible were characterized by almost symmetrical during inclination and amplitude at the right and left articular pathway. In the 2 nd group of patients with dentition defects and deformations we established the following qualitative characteristics of the condyle displacements. In the examined group of patients there were differences of the qualitative indicators in comparison with the control group. Excursion and incursion movements did not overlap, indicating to the dysfunction of the masticatory muscles and the presence of a clinically pronounced syndrome of TMJ dysfunction.

The qualitative condition of the reproducible movements is average during protrusion and vertical movements. The biggest part of patients had a violation of the articular pathway symmetry but protrusion movements are symmetrical during inclination to the right and left, despite the amplitude is asymmetric. At all patients of the 1st group we indicate amplitude shortening of the sagital articular path during protrusion and vertical movements. However, at patients of this group, 6 and 12 months after treatment with a help of a condylography we indicate a positive dynamic of the qualitative parameters of articular heads movements. Excursion and incursion movements overlapped at $75 \%$ of patients. The qualitative state of reproducible movements remained at average level during protrusion, vertical and transverse movements. Protrusion movements during the slope remain symmetrical and amplitude remains more symmetrical than before treatment according to the condylography. The magnitude of mediotrusion movements is much better after treatment what indicates to the gradual comeback of a symmetrical transverse movements and the absence of a mandibular movement blockage. Furthermore, the improvement of TMJ biomechanical parameters was the evidence for amplitude increase and joint symmetry during vertical mandible movements.

\section{CONCLUSIONS}

The results of clinical observations and the study of TMJ individual parameters with a help of "Cadiax Compact" prove the high efficiency of the proposed comprehensive treatment at patients with dentition defects and TMJ dysfunction, which includes the usage of the proposed tire to stabilize the position of TMJ articular heads.

Proposed by us complex treatment of TMJ functional disorders is carried out in stages and includes the diagnosis of occlusal relations, selective grinding of occlusal surfaces, conduction of the condylography with a help of the system "Cadiax Compact", the usage of an aqualizer for 3-4 weeks and manufacturing of the individual tire for 3-6 months. After eliminating all the manifestations of TMJ dysfunction we performed permanent prosthetics with aesthetic non-removable dentures. The proposed design of the individual tire makes it possible to gradually and gradually increase the bite height and individually form the occlusal ratios and thickness of the tire, considering the position of condylar head of mandible and location of dentition defects. After the proposed treatment, 6 and 12 months later we found that indicators of vertical and protrusion movements of the articular heads at patients of 2nd group were significantly better than indicators at 1st group, who received conventional treatment. According to the results of the mandible transverse movements at patients of 2 nd group after the usage of an individual tire the indicators has significantly increased $(\mathrm{p}<0,05)$ - up to $8,1 \pm 0,3 \mathrm{~mm}$ and 7,8 $\pm 0,4 \mathrm{~mm}$, respectively. Moreover, in 6 and 12 months after prosthetics it is possible to note stabilization and improvement of these indicators in comparison with the previous data.

\section{REFERENCES}

1. Rozhko MM, Nespryadko VP. Prosthetic dentistry. K.: Knyga plus.2003;552 (in Ukrainian).

2. Briggs KA, Breik 0 , Ito K, Goss AN. Arthrocentesis in the management of internal derangement of the temporomandibular joint, Australian Dental Journal,2018; 64(1):90-95.

3. Tkachuk OE, Sysolyatin SP, Popova TR. Manifestations features during the study of the prevalence of internal TMJ disorders at people aged from 8 to 22 years: materials of the Republican scientific and practical conference "Achievement of future progress in the field of dental surgery - the Department of Surgical Dentistry and Facial Surgery of KNMU". Kharkiv.2010;100-101.

4. Shiyan VP. The prevalence of the TMJ functional disorders at people of different ages: materials of Ukrainian practical-scientific conference with international participants"Successful prospect of the dental surgery and the facial surgery development". Kharkiv.2010;104-106.

5. Slade GD, Ohrbach R, Greenspan JD. Painful temporomandibular disorder: Decade of discovery from OPPERA studies. J Dent Res.2016;95:10841092.

6. Makjejev VF, Kulinchenko RV. Features of patients examination with temporo-mandibular joint disorders. Ukrains'kyj stomatologichnyj almanah. 2006;1(1):53-61 (in Ukrainian).

7. Slavicek R. Relationship between occlusion and temporomandibular joint disorders: implications for the gnatologist. Am J Orthod Dentofacial Orthop. 2011 Jan;139(1):10,12,14 passim. doi: 10.1016/j. ajodo.2010.11.011.

8. Landa JS. Study of the temporomandibular joint syndrome. Ann Dent. 2015;9:5-14.

9. Campbell J.Distribution and treatment of pain during temporomandibular arthrosis. Brit. Dent. J.2018;105:393-398.

10. Buman A, Lotzmann U. Anatomy of the masticatory system. In: TMJ disorders and orofacial pain: the role of dentistry in a multidisciplinary approach (color atlas of dental medicine). Thieme Medical Publishing.2002;11-52.

11. Xiang GL, Long X, Deng MH, Han QC, Meng QG, Li B. A retrospective study of temporomandibular joint ankylosis secondary to surgical treatment of mandibular condylar fractures. British Journal of Oral and Maxillofacial Surgery.2014;3:270-274. 
12. Kreiner M, Okeson J, Tanco V, Waldenström A, Isberg A. Orofacial Pain and Toothache as the Sole Symptom of an Acute Myocardial Infarction Entails as a Major Risk of Misdiagnosis and Death. J Oral Facial Pain Headache.2019 doi: 10.11607/ofph.2480.

13. Moreno-Hay I, Okeson JP. Single event versus recurrent luxation of the temporomandibular joint. J Am Dent Assoc.2019;150(3):225-229.

14. Jeffrey P. Okeson. Management of temporomandibular disorders and occlusion. 7th edition. Mosby.2012;504.

15. Robert M.Conville, Faye Moriarty, Simon Atkins. The management of temporomandibular disorders: a headache in general practice, British Journal of General Practice.2019;687:523-524.

16. Ribalov OV, Moskalenko PA, Semenenko YI. The results of the biopotentials study of the masticatory muscles at patients with varying degrees of hypermobility of the temporomandibular joint. World of Medicine and Biology.2011;1:91-94 (in Ukrainian).

17. Volovar OS, Kostyuk TM, Litovchenko NM. Study of the neuromuscular disorders at patients with temporomandibular joint dysfunction: Materials of Ukrainian practical-scientific conference "New technologies in dentistry and maxillofacial surgery". Kharkiv.2006;153-155.

18. Novikov VM. Record of electromyographic studies of the reflexadaptation processes during functional disorders of the dental-maxillary system. Magasine of problems of biology and medicine. 2016;4:96-99.

19. Schiffman E, Ohrbach R, Truelove E. Diagnostic criteria for temporomandibular disorders (DC/TMD) for clinical and research applications: recommendations of the International RDC/TMD Consortium Network and Orofacial Pain Special Interest Group. J Oral Facial Pain Headache. 2014;28:6-27.

20. BabichVV, Ryzhak GA., Iordanishvili AK. Explanation of some physiological characteristics of homeostasis at elderly patients with temporomandibular joint dysfunction. Adv. Gerontol. 2014;27(3):575-577.

21. Shetty R. Prevalence of Signs of Temporomandibular Joint Dysfunction in Asymptomatic Edentulous Subjects: A Cross-Sectional Study. The Journal of Indian Prosthodontic Society. 2008; (10)2:96-101.

22. Smaglyuk LV. Functional state of the masticatory muscles combined with occlusion pathology of the Engle 2nd class and TMJ dysfunction. Ukrainian Dental Almanah.2004;3:59-62 (in Ukrainian).

23. Khvatova VA, Chikunov S0. Occlusal tires. M.: Medical book.2010; 153 (in Russian).
24. Novikov VM, Lunkova YS. Correlation between musculoskeletal dysfunction of the TMJ and occlusal disorders in different types of occlusion. Problems of ecology and medicine.2011;15(3-4):20-122.

The relationship of the publication to the planned research work: Within the research work of the Prosthetic Dentistry Department of Ivano-Frankivsk National Medical University "Clinical and experimental substantiation of improving the quality of prosthetic treatment at patients with dental pathology" (state registration №0112U000573). The applicant is a co-executor of a scientific work fragment.

\section{ORCID and contributionship:}

Roman Ozhohan: 0000-0003-4220-2658 $8^{C, D, E}$

Mykola M. Rozhko: 0000-0002-6876-2533 ${ }^{A, E, F}$

Zinoviy R. Ozhogan: 0000-0003-2759-2926

Roman M. Khopta: 0000-0001-5721-9060

Lidiia Miziuk: 0000-0002-6333-525X $X^{B, D}$

\section{Conflict of interest:}

The Authors declare no conflict of interest

\section{CORRESPONDING AUTHOR Roman 0zhohan \\ Ivano-Frankivsk National Medical University \\ Ivano-Frankivsk, Ukraine \\ e-mail:helennochka@i.ua}

Received: 11.06 .2020

Accepted: 01.09 .2020

A - Work concept and design, B - Data collection and analysis, C - Responsibility for statistical analysis, D -Writing the article, $\mathbf{E}$-Critical review, $\mathbf{F}$ - Final approval of the article 